\title{
Del urbanismo androcéntrico a la ciudad cuidadora
}

\author{
From the androcentric urbanism to the caring city
}

\author{
Blanca Valdivia ${ }^{1}$ \\ Fecha de recepción: 08-04-2018 - Fecha de aceptación: 07-08-2018 \\ Hábitat y Sociedad (ISSN 2173-125X), n..$^{\circ} 11$, noviembre de 2018, pp. 65-84. \\ http://dx.doi.org/10.12795/HabitatySociedad.2018.i11.05
}

\section{Summary}

The urban configuration is not neutral. In urban planning and design, the development of certain activities is prioritized while others are marginal and are expected to be solved by themselves.

From the Industrial Revolution begins to specialize spaces according to the activities that were developed in them. The public sphere was associated with the productive and the private sphere with the reproductive sphere, cementing this separation from the sexual division of labor that also leads to a segregation of spaces according to the sexes.

The allocation of reproductive activities to the domestic space has led to our current cities are not designed to meet the care, which negatively affects the quality of life and the daily lives of people who develop these activities, which remain mostly women,

In order to have a fairer and more equitable society, it is necessary to make a change in the urban paradigm and begin to build the caretaker city, in which the sustainability of life is at the center of urban decisions.

\section{Key words}

Caring city; Feminist Urban Planning; Gender

\section{Resumen}

La configuración urbana no es neutra. En la planificación y el diseño urbano se prioriza el desarrollo de determinadas actividades mientras que otras son marginales y se espera que se resuelvan por sí solas.

A partir de la Revolución Industrial comienza a darse una especialización de los espacios según las actividades que se desarrollaban en ellos. Se asociaba el ámbito público con lo productivo y el ámbito privado con la esfera reproductiva, cimentando esta separación a partir de la división sexual del trabajo que lleva también a una segregación de los espacios según los sexos.

La asignación de las actividades reproductivas al espacio doméstico ha llevado a que nuestras ciudades actuales no estén pensadas para satisfacer los cuidados, lo que incide negativamente en la calidad de vida y en la vida cotidiana de las personas que desarrollan estas actividades, que siguen siendo mayoritariamente mujeres.

Para tener una sociedad más justa y equitativa es necesario hacer un cambio de paradigma urbano y comenzar a construir la ciudad cuidadora, en la que la sostenibilidad de la vida está en el centro de las decisiones urbanas.

\section{Pallabras clave}

Ciudad cuidadora; Urbanismo Feminista; Género

1 Socióloga y doctoranda en la ETSAB (Barcelona); desde 2009 es socia colaboradora de la cooperativa Col.lectiu Punt 6. E-mail: blanca.valdivia@gmail.com. 


\section{Introducción}

El sistema patriarcal como conjunto de normas y valores dominantes en la sociedad influye en todas las esferas y ámbitos de la sociedad y también en la producción del espacio. Jane Darke (1998a) señala que el patriarcado adopta muchas formas y cambia con el tiempo. Coexiste con la mayoría de los sistemas económicos, incluido el capitalismo, y en muchos escenarios: en la familia, en el lugar de trabajo, en el gobierno, etc. Está tan profundamente arraigado en las relaciones sociales que mucha gente no lo identifica y considera la dominación masculina como algo natural (ob. cit.).

La configuración espacial reproduce la dicotomía público y privado y la división sexual del trabajo, pero, al mismo tiempo, el espacio reproduce y contribuye a la propagación de dichos dualismos. El desarrollo de la ciudad moderna se sustenta en este dualismo según el cual a cada espacio se le atribuyen unas funciones y actividades concretas y donde las actividades productivas son priorizadas en el diseño urbano, invisibilizando las necesidades de la esfera reproductiva.

\section{La división sexual del espacio}

El dualismo público-privado configura el espacio segregándolo según estas dos esferas y le asigna funciones específicas (productivo-reproductivo), a las que también se le atribuyen categorías genéricas (masculino-femenino). Sin embargo, esta dicotomía no ha sido una constante histórica, sino que tiene su origen en los inicios del sistema capitalista y es una consecuencia de la división sexual del trabajo.

Esta división llevó a una delimitación de ámbitos espaciales masculinos y femeninos sobre los cuales se proyectó una serie de valores e ideologías que han reforzado la construcción cultural de las categorías hombre y mujer (Fernández, 1995).

Esta división sexual del trabajo iría acompañada de unos determinados roles asignados a cada sexo. Según M ${ }^{a}$ Ángeles Durán (1998), con la división sexual del trabajo, enmarcada en el seno de la familia, los hombres se encargan de las tareas productivas, las relacionadas con el mercado, que se dan en el ámbito de lo público, mientras que las mujeres son las encargadas de las tareas reproductivas, que se dan en el ámbito de lo domestico. Bourdieu (2000) señala que la dominación masculina se apoya en la división sexual de trabajo que asigna tareas concretas a cada uno de los sexos y establece una oposición entre el lugar de reunión o mercado, reservado a los hombres, y la casa, reservada a las mujeres. La diferencia biológica se utiliza como justificación natural de la diferencia construida socialmente entre los sexos y de la división sexual del trabajo (ob. cit.).

Carrasco, Borderias y Torns (2011) señalan que los hogares preindustriales aunaban funciones productivas y reproductivas y que la división sexual del trabajo mercantil y doméstico y de cuidados variaba bastante según los contextos económicos. La comunidad doméstica albergaba en el mismo espacio la producción artesanal y la habitabilidad del hogar, es decir, los trabajos realizados dentro de la unidad familiar más los salarios aportados por sus miembros (Murillo, 1996).

Las sociedades preindustriales europeas se caracterizaban por la unión de la esfera productiva y reproductiva en una forma de vida en las aldeas comunales. Con el surgir del capitalismo la esfera reproduc- 
tiva fue sacada de la esfera comunitaria y relegada a lo privado, aislándola a la esfera del hogar, mientras que la producción de mercancías aumentó y se trasladó a las fábricas para una producción a mayor escala en el marco del sistema de trabajo asalariado (England, 1991).

Con el progreso de la Revolución industrial surge la figura del trabajador fabril que sale de casa para desplazarse hasta un centro de producción. El espacio doméstico se vuelve un espacio secundario y deja de tener la capacidad de generar productos de subsistencia (que pasan a adquirirse en el mercado), perdiendo valor y control sobre el nuevo concepto de trabajo, que se vuelve indisociable del salario (Murillo, 1996).

El proceso de desarrollo del capitalismo industrial que acarreó la separación entre hogar y trabajo y basado en las distinciones funcionales y biológicas entre mujeres y hombres, promovía la división de tareas como fórmula más eficiente y productiva para organizar el trabajo, los negocios y la vida social (Grupo de Ecofeminismo -Ecologistas en Acción, 2011). La idea liberal y burguesa de familia que sitúa al padre como sustentador económico y a la madre como ama de casa se fue instituyendo como el modelo en el discurso dominante (Brullet, 2010).

La construcción y consolidación de la sociedad industrial que situó a las mujeres en el espacio privado, con la función principal de cuidar de los otros en la vida diaria en una situación de subordinación social, política y económica, se apoyó no sólo en la institución de la familia moderna sino también del resto de estructuras institucionales modernas (sistema jurídico, organización de los espacios y del tiempo social, relaciones laborales, escuela, empresas, sindicatos, gobiernos políticos, etc.) (ob. cit.).

El inició de la Revolución industrial no solo llevó a la separación del espacio público-privado y a la identificación de lo masculino-femenino y lo productivo-reproductivo con cada uno de los espacios, sino que también derivó en lo que Carrasco, Borderias y Torns (2011) señalan como la construcción social de la desvalorización de los trabajos domésticos y de cuidados que acompañó al desarrollo de la producción mercantil.

La reproducción implica permitirle al otro subsistir, física y afectivamente. Sin embargo, mientras que en lo productivo recaen el prestigio, la autonomía y el poder de decisión, la reproducción y su práctica diaria le ha rebajado a la categoría de rutina, y por definición, no reporta nada extraordinario. Lo productivo va unido a las actividades públicas mientras que lo reproductivo queda imbuido en el ámbito doméstico y se conforma el dominio de lo productivo sobre el reproductivo (Murillo, 1996).

Carrasco, Borderias y Torns (2011) identifican durante la industrialización un cambio radical en el modelo de división sexual del trabajo y nuevas identidades de género, habiendo un desplazamiento de los cuidados desde el servicio doméstico o la comunidad al ámbito privado y de la familia, y de las redes femeninas de cuidados, asalariados o no, a la madre, y cómo este fue un proceso lento y difícil especialmente entre las clases trabajadoras debido a las altas tasas de actividad femenina de la época. También se dieron profundos y complejos cambios en las características y condiciones del trabajo familiar doméstico entre los que se encuentran la transformación en la concepción de la maternidad, el nuevo valor dado a la infancia y a los trabajos de cuidados de niños y niñas, personas ancianas y enfermas, y también los cuidados a los hombres sustentadores económicos, quienes tenían una dedicación completa al trabajo de mercado (que a mediados del siglo XIX podía 
llegar a las setenta y dos horas semanales) siendo esta dedicación sostenida por el trabajo de reproducción cotidiana de las mujeres de sus familias (ob. cit.).

¿Pero qué repercusión tuvo la división sexual del trabajo sobre la construcción urbana durante esta época? Según McDowell (1996) el modelo de ciudad del siglo XX es la concreción urbana de la separación del puesto de trabajo y la casa, la ciudad y la periferia, la vida pública y la vida privada y familiar que supuso la Revolución Industrial en occidente. Esta segregación espacial se basaba en dos esferas excluyentes de actividades, la productiva y la reproductiva. La esfera productiva se identificaba con el espacio público, y es el espacio asignado a los hombres y donde se desarrollaban las actividades económicas, políticas, culturales, etc., mientras que la esfera reproductiva se situaba en el espacio privado o doméstico, al que eran relegadas las mujeres.

En las sociedades capitalistas del Norte global, el modelo de reparto de los cuidados, que asigna a las mujeres las tareas de cuidadoras y a los hombres el trabajo asalariado, ha atravesado no sólo la estructura de hogares y del mercado laboral, sino también las políticas públicas y la propia construcción de las identidades (Agenjo, 2013).

El dividir los espacios en público y privado y asignarle a cada uno una responsabilidad masculina o femenina tiene consecuencias discriminadoras y atenta contra la igualdad de oportunidades, ya que la liberación de un tiempo doméstico es fundamental para tener un tiempo en el que dedicarse a lo que uno desee y la posibilidad de construir una individualidad. Esta falta de privacidad provoca una posición deficitaria en el espacio público (Murillo, 1996).

La Revolución Industrial es el momento en que comienza a identificarse a las mujeres con el espacio doméstico y también cuando comienza a construirse la concepción social del espacio público como espacio ajeno e inapropiado para las mujeres.

Los espacios surgen de las relaciones de poder, las relaciones de poder establecen las normas; y las normas definen los límites tanto sociales como espaciales, determinan quién pertenece a un lugar y quien queda excluido y dónde se localiza una determinada experiencia (McDowell, 1999).

La exclusión de las mujeres del ámbito público se apoya en la división sexual de los trabajos y de los espacios y se materializa en una configuración de los espacios centrada en las experiencias y necesidades masculinas.

Sandercorck y Forsyth (1992) señalan que en planificación urbana la línea establecida entre lo público y lo privado, o la vida doméstica, se ha configurado poniendo en una situación de ventaja a los hombres. Por tanto, la dimensión pública es una construcción física que por definición representa toda una serie de cuestiones políticas y económicas disputadas en el marco de la planificación.

La conceptualización de los espacios a partir de las exclusiones de actividades y sexos continua en la actualidad. Sin embargo es importante romper con este enfoque dualista de los espacios privados y públicos, ya que por una parte sitúa determinadas experiencias y actividades en un espacio mientras que las excluye del otro y, por otra parte, perpetúa la idea de que las cosas públicas son de responsabilidad común, pública, comunitaria, mientras que las cosas que pasan en el ámbito doméstico se quedan en el ámbito de lo privado y por lo tanto se siguen reproduciendo jerarquías y desigualdades basadas en el género. 
Continuar pensando los espacios desde la dicotomía público-privado invisibiliza la contribución de las mujeres en la actividad económica, política y cultural, así como su participación en el espacio y la esfera pública. A pesar de que ha habido una invisibilización histórica de las mujeres en la esfera pública, las mujeres participaron activamente de la Revolución Industrial como trabajadoras ya que, como nos recuerda Isabel Segura (2006), el proceso de industrialización se hace con la mano de obra de las mujeres tanto en el papel de trabajadora asalariada como de trabajadora en el espacio doméstico, siendo la responsable del cuidado de las personas de la familia, pero sin ningún tipo de retribución. Las mujeres siempre han estado presentes en los espacios públicos de las ciudades, comprando y vendiendo mercancías, caminando por las calles para ir a trabajar y participando en celebraciones religiosas y civiles (Ryan, 1990).

La concepción de las mujeres aisladas de la esfera pública es una visión muy reduccionista, ya que como señala Agenjo (2013) comienza a cuestionarse la existencia del modelo de actividades y espacios asignados de manera exclusiva a los géneros más allá de los hogares burgueses y se considera como una visión mitificada de tinte burgués. Esta visión además de tener principalmente en cuenta a las mujeres burguesas es profundamente eurocéntrica, ya que responde a los patrones sociales de un momento histórico, a partir de la Revolución Industrial, de clases sociales determinadas y de un contexto geográfico específico, Europa y EEUU.

Por último, es imprescindible recoger también el legado de las mujeres en la configuración del espacio urbano. La ciudad industrial antepone los criterios productivistas en su configuración, pero mujeres de todo tipo intervinieron activamente en la demanda de mejores condiciones de salubridad e higiene en las ciudades, enfrentándose o colaborando con las autoridades municipales (Velázquez, 2006).

La naturalización del trabajo de cuidados y la identificación de estas tareas con lo femenino, llevó a una desvalorización de las mismas y a relegar estas actividades (conceptualmente) al espacio doméstico, a pesar de que hay un gran número de actividades de cuidados que se desarrollan en el espacio público (hacer la compra, cuidar a la infancia, acompañar personas mayores a centros de salud...). Esto ha provocado que los espacios urbanos se hayan pensado desde las necesidades de la esfera productiva pero que no se haya tenido en cuenta en el diseño de los espacios la satisfacción de las necesidades vinculadas con la esfera reproductiva. Es decir, la ciudad no se ha pensado como el soporte físico para poder desarrollar las actividades de cuidados, ya que desde la Revolución Industrial se ha considerado que las actividades de cuidados se llevaban a cabo exclusivamente en el espacio doméstico y por las mujeres.

\section{La ciudad capitalista y patriarcal}

La división del trabajo en el marco del sistema social patriarcal fue la base a partir de la cual comienzan a conformarse las ciudades. La configuración espacial sirvió al mismo tiempo como reproductora de las estructuras del sistema. Los diferentes paradigmas urbanísticos de los que son herederas nuestras ciudades actuales se basan en esta concepción socio-espacial.

El discurso social delimita los distintos usos de los espacios y la distribución de los lugares, y asigna protagonismos, dependiendo del gé- 
nero de sus habitantes. El espacio público será gestionado mayoritariamente por hombres mientras que el espacio doméstico tendrá a las mujeres como máximas responsables (Murillo, 1996).

Jane Darke (1998a) defiende que en todo asentamiento las relaciones sociales de la sociedad que lo ha construido se inscriben en el espacio, quedando las funciones y el lugar apropiado para las diferentes categorías de personas (de género, grupos de edad, castas, clases o grupos étnicos) incorporados a pueblos y ciudades. Nuestras ciudades están embebidas de patriarcado, presente en la piedra, el ladrillo, el vidrio y el hormigón. Existe toda una serie de normas implícitas y explícitas que establecen cuáles son los cuerpos que pueden acceder a ciertos espacios y cómo se relacionan entre sí. La normatividad androcéntrica se plasma y transmite no solo en la configuración urbana sino también en la forma de los edificios, tanto en sus divisiones interiores como en los espacios que los separan (McDowell, 1999).

La configuración de las ciudades responde a una concepción de la vida cotidiana cimentada en la división sexual del trabajo y que se basa en una dicotomía artificial de lo público/privado y masculino/femenino (Murillo, 1996; McDowell, 1999; Durán, 2000; Sánchez de Madariaga, 2004; Muxí Martínez, 2009). Estas formas convencionales del diseño, legado de los principios del movimiento moderno y basadas en la división sexual del trabajo, asumen la existencia en cada hogar de una persona que se ocupa a nivel individual del cuidado de las personas dependientes y de las múltiples tareas necesarias para el mantenimiento de la vida cotidiana. De esta concepción dicotómica de la esfera pública y la esfera privada y del trabajo productivo frente al trabajo reproductivo derivan las decisiones sobre la forma de la ciudad, la distribución de usos en el espacio, las inversiones públicas en infraestructuras y transporte, la concepción de los espacios domésticos, etc., que contribuyen a potenciar el funcionamiento del sistema productivo más que a facilitar las tareas necesarias para la reproducción social y la organización de la vida cotidiana (Sánchez de Madariaga, 2004). Muxí Martínez (2009) relaciona la existencia de dos esferas de trabajo, una remunerada y reconocida y otra no remunerada e invisible -que se corresponden con una división sexual del trabajo- con la ciudad por partes, la ciudad de las funciones segregadas que ha degenerado en una ciudad triplemente segregada por funciones, clase y género. Frente a esta realidad las mujeres reclaman una ciudad compleja y de proximidad, con buen transporte público y espacios públicos seguros que permitan elegir el uso que se hace de la ciudad.

Según Darke (1998b) en la ciudad siempre ha habido espacios diferenciados pero este fenómeno se agudiza con la ciudad zonificada a partir del s. XIX, con la industrialización y la urbanización acelerada que cambiaron radicalmente la sociedad. En el siglo XIX los roles de género estuvieron mucho más diferenciados y simultáneamente las ciudades adquirieron mayor complejidad espacial, con la separación de funciones y el inicio de la suburbanización a gran escala.

La polarización de los espacios público y privado a partir de la división sexual del trabajo continúa con la expansión del modelo de suburbio americano, que además ha sido extendido y replicado en todo el mundo hasta nuestros días. Dolores Hayden (1982) critica este modelo de crecimiento urbano que se sustenta en la división sexual del trabajo y que convierte el espacio residencial en una jaula de oro para las amas de casa, apartándolas de nuevo de la esfera productiva donde habían tenido un papel protagonista durante la Segunda Guerra Mundial. 
El discurso dominante de la posguerra reconvierte el hogar y el cuidado familiar en la única aspiración posible para las mujeres: la cocina como fábrica, la casa amplia y moderna, unos hijos sanos y felices y un marido exitoso que trabajara en las corporaciones situadas en la ciudad. El éxito para las mujeres consistía en ser una feliz ama de casa. La mística de la feminidad, de Betty Friedan de 1963, es una crítica a partir de su propia experiencia de este sentimiento de insatisfacción compartido con otras mujeres que condujo a una depresión generalizada entre las mujeres en la década de 1950-1960 y que los médicos denominaron la enfermedad sin nombre (Muxí Martínez, 2013).

Las ciudades de posguerra priorizaron el empleo masculino, construidas a partir de la separación de ámbitos y basada en los roles tradicionales, pero sin tener en cuenta la combinación de roles de esposa, madre y trabajadora de muchas mujeres. El empleo de las mujeres a tiempo parcial (permitiendo la conciliación con las responsabilidades familiares) era difícil debido al escaso transporte público a las zonas industriales fuera de las horas punta. Las zonas residenciales fueron diseñadas sobre la concepción tradicional según la cual el hombre trabaja fuera de casa para ganar un dinero que cubra el sustento familiar, y la esposa cumple el papel de ama de casa sin ocupación remunerada. Elementos de la configuración de este modelo urbano, como la falta de equipamientos para el cuidado de las criaturas y de redes familiares locales, la ausencia de comercios en las proximidades y los recorridos largos y caros hasta el centro de trabajo, impedían prácticamente el desempeño de otros papeles por parte de las mujeres (Darke, 1998b).

Decenios dominados por un enfoque sectorial en la planificación urbanística nos han conducido a entornos urbanos segregados donde los ambientes residenciales, de trabajo, compras y ocio constituyen esferas independientes unidas por extensos sistemas de transportes (Jaeckel y Van Geldermalsen, 2006). Este modelo territorial dificulta el desarrollo de la vida cotidiana e impide que exista una conciliación entre las diferentes actividades que se desarrollan en el día a día.

La ciudad zonificada segrega actividades cotidianas como el trabajo, el ocio, la movilidad y la vida familiar, pero la mayoría de las mujeres no separan de esa manera la realización de estas actividades (Darke, 1998b). De hecho, según Carrasco Bengoa (2007) el feminismo, que se basa en la experiencia de las mujeres, pone en evidencia que en la vida humana no existen compartimentos estancos porque la realidad es mucho más fluida y se caracteriza por un conjunto de espacios interrelacionados (privado, personal, doméstico, público...). La vida de las mujeres es un constante transitar entre los diversos espacios privados y los diversos espacios públicos en un continuo de experiencias que confirma la permeabilidad de los espacios, y todavía más allá, cuestiona que se trate de espacios delimitados, independientes el uno del otro. (ob. cit.).

Pero si en el marco de la ciudad capitalista se construyó una ciudad hecha a la medida del hombre no ocurrió algo muy diferente en la conformación de las ciudades comunistas. En la construcción de la ciudad socialista tuvieron mucha relevancia los 16 principios del urbanismo de la RDA, denominada la "Anti-Carta de Atenas", presentada en la CIAM de Bérgamo en 1949. En estos principios se decía que la ciudad no puede ser construida como suma casual de barrios y que el objetivo del urbanismo es la satisfacción de los derechos humanos al trabajo, la vivienda, la cultura y el ocio (Sáinz Guerra, 2004). Además, en el tercero de estos principios se establece la industria como esencia de las acti- 
vidades urbanas (ob. cit.). Tal como pasa en la Carta de Atenas con las funciones urbanas, se olvidan de las respuestas que tiene que dar la ciudad a las necesidades derivadas de la esfera reproductiva y los cuidados.

Además de los diferentes paradigmas urbanos que han dejado su impronta sobre la conformación de la ciudad actual, las políticas neoliberales, y los recortes en épocas más recientes, han provocado grandes desequilibrios sociales que se concretan territorialmente en fenómenos como la mercantilización del espacio público, la especulación, la gentrificación y/o la turistificación.

En esta ciudad social y económicamente injusta las características sociales como el género, la clase social, el ser una persona racializada, la identidad sexual, la diversidad funcional o la edad, entre otros aspectos, determinan los privilegios y las opresiones que experimentamos en nuestro día a día en el espacio urbano.

Como respuesta a las dificultades para el desarrollo de la vida cotidiana y analizando los diferentes usos que mujeres y hombres hacen del espacio urbano, según las tareas que desarrollan en su día a día, las geógrafas feministas comenzaron a estudiar en los años 70 el entorno urbano desde una perspectiva de género (García Ramón, 1989, 2005; Sabaté el al., 1995; McDowell 1999; Bondi y Rose, 2003) estableciendo una relación entre los roles de género y las divisiones espaciales. Las geógrafas feministas se plantean hasta qué punto los hombres y las mujeres viven de manera diferenciada los espacios y los lugares, y pretenden demostrar que estas diferencias forman parte tanto de la constitución social del espacio como del género.

Muchas autoras desde la perspectiva de género han remarcado la existencia de pluralidad de necesidades y los problemas que conlleva no tener en cuenta esta diversidad. Siguiendo esta argumentación, muchas autoras que han hecho una lectura desde el territorio, han denunciado el carácter androcéntrico de las ciudades al invisibilizarse las necesidades relacionadas con las tareas reproductivas y diseñarse los espacios sin tener en cuenta la vida cotidiana de las mujeres (Moser y Levy, 1986; Moser, 1989; Campos, 1996; Levy, 1996, 2003; Walker et al., 2013).

Para Beall (2010) mujeres y hombres tienen diferentes intereses y necesidades en las distintas etapas de sus vidas y estas van variando también según los diversos contextos familiares y comunitarios. Las ciudades como expresión espacial de las relaciones sociales están basadas en el poder y el conflicto y también en la cooperación y el consenso, lo que ha significado que muchas veces las necesidades de las mujeres hayan sido ignoradas.

A partir de la diversidad de experiencias, la vida cotidiana de mujeres y hombres es diferente. Las actividades desarrolladas en el día a día y la gestión del tiempo están marcados por los roles de género y el hecho de tener (o no) un cuerpo sexuado. Por otro lado, las personas desarrollan su vida cotidiana en una estructura urbana que se ha diseñado desde una perspectiva androcéntrica. Determinadas actividades son consideradas socialmente más importantes y esto se materializa en una configuración urbana que prioriza unas actividades y jerarquiza unos usos frente a otros, dedicándoles más espacio, mejores localizaciones, conectividad... Estos factores afectan al desarrollo de unas y otras actividades.

Según Clara Greed (1997) las mujeres hacen un uso diferente de la ciudad a los hombres, y que esto se debe a que tienen responsabilidades y roles distintos. Además, se toma a los hombres como modelo, 
como el "ser humano medio" y el urbanismo se enfoca en satisfacer estas necesidades. La autora también defiende que "la planificación urbanística puede ser cualquier cosa que queramos, no es algo que está prefijado, no es un don de Dios, es una creación de realidades para mujeres y para hombres" (ob. cit., p. 2).

Soto Villagrán (2007) señala que desde el feminismo se cuestiona la distribución desigual de los espacios y la asignación diferencial de las esferas doméstica y pública, ya que en los espacios donde se lleva a cabo la vida cotidiana, la movilidad y las actividades de las mujeres responden a estereotipos femeninos, influidos por una perspectiva masculina tanto de la planificación como de la cultura dominante.

Derivados del tipo de tareas que desempeñan en su día a día, mujeres y hombres hacen un uso diferente del espacio urbano. Como cuestiones generales se podría señalar que las mujeres utilizan más el transporte público y hacen más recorridos a pie (Miralles-Guasch, 2010). El problema con el que se enfrentan las mujeres en cuanto a la movilidad es que los sistemas de transporte han sido diseñados a partir de la jornada laboral masculina y por eso el foco sobre la planificación en transporte se ha puesto sobre la movilidad en lugar de en la accesibilidad (Beall,1996). Sánchez de Madariaga (2004) afirma que, a pesar de las diferencias entre barrios, ciudades y países, existen determinadas características generales sobre la movilidad femenina, como patrones de movilidad más complejos fruto de sus múltiples responsabilidades y un menor acceso al vehículo privado. Además, realizan movimientos poligonales (a diferencia de los hombres que los hacen pendulares), son las principales usuarias del transporte público, encadenan más viajes, viajan por mayor variedad de motivos, hacen recorridos más cortos -y gran parte de los desplazamientos son en el entorno residencial- y hacen muchos viajes acompañando a otras personas que carecen de autonomía personal. Aunque se mueven más, su movilidad está limitada por la dependencia de las necesidades de otras personas y de los horarios de los servicios públicos. Esta reducción de su movilidad afecta a su capacidad de acceso al empleo y a otras actividades como el ocio. También utilizan más ciertos equipamientos o servicios, al asumir en mayor medida tareas relacionadas con los cuidados y la esfera reproductiva, como servicios médicos, espacios públicos (como acompañantes y cuidadoras de niños y niñas) y mercados o tiendas (Llop, 1996).

Pascuala Campos (1996) alerta de que en múltiples ocasiones las necesidades que conllevan los trabajos de la vida cotidiana son olvidadas, por eso es importante visibilizar estas tareas y evidenciar el rol imprescindible que desempeñan para el mantenimiento de cualquier sociedad. Las actividades relacionadas con la atención y el cuidado de los niños y niñas, de las personas mayores y enfermas, y de todos los trabajos relativos a la higiene, alimentación y atención afectiva son considerados resolubles de una manera "natural", es decir resueltos en la mayoría de los casos por mujeres (ob. cit.). A efectos prácticos esto implica que en la mayoría de las ocasiones no son tenidos en cuenta a la hora de planificar el territorio y de hacer políticas públicas.

Clara Greed (1997) recoge algunos temas que afectan negativamente a las mujeres, en relación a las actividades que hacen en su día a día y por cómo se materializa su vida cotidiana en el espacio físico, y clasifica estos problemas según las diferentes escalas del territorio. En la escala de lo "macro" y de la planificación urbanística los problemas que se encuentran están vinculados con la zonificación segregada, escasez de transporte público y descentralización del comercio; en la escala de lo 
"meso", del distrito, la falta de equipamientos, tiendas y oportunidades de empleo; y en la escala local, de lo "micro", los temas de seguridad y los problemas derivados de la accesibilidad y el cuidado de los hijos.

Según Teresa del Valle (1996) la construcción del espacio urbano está más orientada a mantener a las mujeres en los espacios destinados a los roles familiares que a promover su incorporación a la sociedad en general.

Larsson (2006) argumenta que el lento avance de la perspectiva de género dentro de la planificación es debido en parte a que la mayoría de los trabajos de ordenación urbana se basan en un género neutro, centrándose en el interés público en general, sin cuestionar el significado de interés público. La autora identifica diferentes factores como causantes de las desigualdades que sigue promoviendo la planificación urbana. Por una parte, la existencia de relaciones de poder y la subordinación de las experiencias de las mujeres en la praxis de la planificación. La manera en que se maneja lo que se considera "ser mujer". El uso de dicotomías como público-privado, reproducción-producción y trabajo remunerado-trabajo no remunerado en relación con los diversos niveles de la planificación. Una comprensión poco clara del concepto de "interés público" y una falta de conocimiento de las actividades de planificación desde la perspectiva de género.

Según Alejandra Massolo (2005), "la perspectiva de género no representa una visión apocalíptica de la ciudad, ni sostiene una concepción "victimista" de las mujeres en la vida urbana, pero sí es una mirada que por su mismo origen en las luchas y los derechos de las mujeres, señala las injustas situaciones existentes, cuestiona que la ciudad sea pensada y organizada a la medida del hombre y pretende cambios que permitan una buena vida de las mujeres, en una ciudad y sociedad más justa y equitativa" (pp. 8-9).

Por su parte Rainero y Rodigou (2001) señalan que existen nuevos patrones en la sociedad que cuestionan esta dicotomía entre lo público y lo privado (nuevas tipologías de trabajo remunerado, flexibles, en el propio hogar; altas tasas de desempleo masculino; mayor protagonismo público de las mujeres; etc.). Estos cambios no se han materializado en una transformación de la organización física de la ciudad y los tiempos de la misma, que acompañe a estas nuevas necesidades, lo que impacta en la calidad de vida de las mujeres que encuentran serias dificultades para la conciliación entre las diferentes esferas (ob. cit.).

Las feministas en las últimas décadas han luchado para aumentar la presencia de mujeres en la vida pública. Durante estos años las feministas han reivindicado los derechos de las mujeres como actores activos en la esfera pública y han reclamado participar plenamente en la vida de la ciudad, crear y proteger espacios para las mujeres y redefinir y extender la definición de esfera pública. Las feministas han argumentado la necesidad de cambios radicales en las estructuras espaciales metropolitanas y cambios en las políticas sociales y de transporte para mejorar las oportunidades de participación en la vida política y económica de la ciudad de las mujeres que además son cuidadoras (Sandercock y Forsyth, 1992).

Werkele (1980) señala que muchos de los trabajos en urbanismo con perspectiva de género apuntan a la necesidad de cambios fundamentales en algunos de los más básicos elementos de la ciudad moderna, como los patrones de zonificación, la planificación de barrios, los sistemas de transporte, la industria de la vivienda y la estructura de servicios sociales. Greed (1997) apunta a que en el nivel micro, de las viviendas y el entorno próximo, las mujeres han expresado durante años su preocupación 
por el tipo de trazado de las calles, las densidades, el diseño de las viviendas y por temas sociales como la delincuencia, la seguridad, el cuidado de niños y niñas, los problemas de tráfico y la accesibilidad.

Jane Darke (1998b) apunta a los diversos trabajos que han analizado cómo el modelo de zonificación se basa en papeles estereotipados según el género, en los cuales las unidades familiares están compuestas por un hombre encargado del sustento económico y con un horario laboral convencional, y una mujer, ama de casa, que utiliza la ciudad de manera diferente, lleva a niñas y niños al colegio, hace las compras... y pasa la mayor parte de su tiempo atendiendo al hogar y a otros miembros de la familia.

Las relaciones y los roles de género son centrales en la localización de recursos, equipamientos y oportunidades en la ciudad, que es esencial en la estructura del espacio urbano. La localización de áreas residenciales, lugares de trabajo, redes de transporte y todas las capas que conforman la ciudad, reflejan las expectativas de una sociedad capitalista y patriarcal sobre qué tipos de actividad tienen lugar dónde, cuándo y por quién. La naturaleza de las relaciones de género se refleja en la estructura espacial de las ciudades, pero igual que las formas de las relaciones de género no son constantes en el tiempo ni en el espacio, la estructura de las ciudades también varía en el tiempo (England, 1991).

Cristina Carrasco (2007) señala algunos factores de planificación urbana que han aumentado las diferencias entre mujeres y hombres, como el desarrollo de un uso funcional del territorio que ha causado un incremento de los desplazamientos en vehículo privado por la extensión de la red viaria interurbana y urbana -además la planificación de la movilidad se ha hecho fundamentalmente para cubrir unas demandas a gran escala y para desplazamientos relacionados con el trabajo o con los estudios, de forma que no se han cubierto necesidades en espacios de menor densidad de población ni para desplazamientos cotidianos no relacionados con el estudio o el trabajo-; el fomento de promociones urbanísticas con viviendas de baja densidad de edificación y pocos servicios de uso cotidiano cerca; el diseño del espacio público desde la perspectiva del coche que ha provocado una pérdida progresiva del espacio destinado a los peatones, y ha creado unas calles inseguras, congestionadas y ruidosas. La vida cotidiana en las calles, por lo tanto, ha ido perdiendo relevancia y ha contribuido a reducir el dinamismo comercial urbano, la localización de equipamientos y servicios públicos, los espacios de juego y de ocio, etc.

Picchio (2009) señala la importancia de incorporar la perspectiva de género para analizar las diferentes experiencias que tienen mujeres y hombres de las condiciones de sostenibilidad de la vida individual y colectiva, ya que podría permitir aclarar mejor las prioridades y los aspectos funcionales de algunos servicios. Este reconocimiento de la diversidad intrínseca en cuanto a la experiencia vital de hombres y mujeres no pretende fijar los roles sexuales, sino abrir un debate público y de negociación social sobre la complejidad del proceso de reproducción y sobre su función en la estructura social (ob. cit.).

\section{Los cuidados en la ciudad}

Las diferencias de género entre mujeres y hombres se manifiestan en las actividades que desarrollan, cómo actúan, cómo utilizan la ciudad, cómo interpretan sus propias vidas y cómo son percibidas socialmente. 
De esta manera, las actividades desarrolladas en el día a día y la gestión del tiempo están marcados por los roles de género y el hecho de tener (o no) un cuerpo sexuado. Teresa Torns señala que la perspectiva de género ha resultado imprescindible para visibilizar dos dimensiones clave de la vida cotidiana: el tiempo y las tareas de reproducción de la vida humana y la relación de estas dimensiones con el bienestar cotidiano (Torns et al., 2006).

La principal diferencia en la vida cotidiana de mujeres y hombres estaría en el tiempo y el grado de responsabilidad dedicado a las tareas de cuidados. En Cataluña, según datos de la Encuesta de usos del tiempo de 2011, las mujeres dedican semanalmente el doble de horas que los hombres a las tareas del hogar y la familia, con un total de 28 horas con 21 minutos, mientras que los hombres dedican 14 horas con 35 minutos.

El cuidado es "una actividad de especie que incluye todo aquello que hacemos para mantener, continuar y reparar nuestro 'mundo' de tal forma que podamos vivir en él lo mejor posible. Ese mundo incluye nuestros cuerpos, nuestros seres y nuestro entorno, todo lo cual buscamos para entretejerlo en una red compleja que sustenta la vida" (Fisher y Tronto 1990, en Tronto 2005, p. 234). Amaia Pérez Orozco (2014) contrapone las actividades de cuidados, que se mueven por una preocupación por la vida ajena a la lógica del capital. Se refiere al conjunto de actividades que, en última instancia, aseguran la vida (humana) y que adquieren sentido en el marco de relaciones interpersonales, gestionando una realidad de interdependencia (ob. cit.). Las personas dependemos física y emocionalmente del tiempo que otras personas nos dan. Durante toda la vida, pero especialmente en ciertos momentos del ciclo vital, sería imposible sobrevivir si no fuese porque otras personas, principalmente mujeres por la división sexual del trabajo, dedican tiempo y energía a cuidarnos. Somos seres encarnados en cuerpos vulnerables que enferman y envejecen y que son contingentes y finitos (Herrero, 2017).

El trabajo de cuidados engloba una notable carga de subjetividad: emociones, sentimientos, afectos-desafectos, amores-desamores. El peligro es que a partir de esta subjetividad se ha construido una identidad femenina en la mística del cuidado basada en los cuidados y en la maternidad. Esto conlleva a que en situaciones duras de cuidados no se cumplan los requisitos de amor que se presuponen y se realizan por la obligación moral socialmente construida que presiona a las mujeres (Carrasco, Borderías y Torns, 2011).

Los trabajos de cuidados producen bienes y servicios para el autoconsumo, no para el intercambio mercantil, generando valores de uso. No persiguen un aumento de la productividad ni fomentan valores de competitividad. Conllevan una fuerte carga emocional (que no siempre tiene por qué ser positiva) y, a diferencia del mercado, responden a una ética que se centra en las relaciones y en las necesidades humanas (Grupo de Ecofeminismo - Ecologistas en Acción, 2011)

El trabajo familiar doméstico que incluye la actividad de cuidados se presenta como un conjunto de necesidades que hay que satisfacer, incluyendo los bienes y servicios directos y las necesidades de afectos, relaciones y cuidados emocionales que se concretan en actividades particulares que, por su carácter subjetivo, pueden ser diferentes para las distintas personas (Bosch, Carrasco y Grau, 2005).

Se pueden clasificar los cuidados en directos e indirectos. Los directos se refieren a actividades realizadas directamente con las personas 
a quien se dirigen los cuidados: dar comida a un bebé, atender a una persona enferma, charlar con una adolescente. Los cuidados indirectos abarcan lo que tradicionalmente se conocía como trabajo doméstico: limpiar la casa, la ropa, cocinar, hacer la compra y también todas las tareas de gestión y organización de los trabajos del hogar (Carrasco, Borderías y Torns, 2011).

Según Amaia Pérez Orozco (2014) es necesario poner la sostenibilidad de la vida en el centro. La posibilidad de alcanzar una vida digna de ser vivida, generando un bien-estar encarnado y cotidiano. La autora critica que la noción hegemónica de vida, que separa vida humana y naturaleza, identifica los valores asociados a la masculinidad con lo humano, impone la irrealidad de la autosuficiencia y asimila bien-estar con consumo mercantil en crecimiento y progreso.

Aunque es imprescindible poner la sostenibilidad de la vida en el centro, es fundamental repensar las connotaciones y significados que tienen los cuidados, por lo que hay que hacer algunas puntualizaciones. Hay que romper con los esencialismos que otorgan a las mujeres unas cualidades especiales para los cuidados a partir de las diferencias biológicas. Situando los cuidados como una actividad social que va más allá de los lazos familiares sanguíneos y superando el modelo de familia nuclear heteronormativa, ya que existen diversidad de modelos familiares que sostienen relaciones afectivas y de cuidados. Visibilizando que, además de los cuidados para la sostenibilidad de la vida, hay parte de los cuidados que suponen un acompañamiento y soporte físico y emocional para la muerte. Poniendo de relieve que frente a las retóricas capitalistas que abogan por un ideal de autosuficiencia todas las personas somos interdependientes de otras personas y además somos dependientes del entorno y del medio ambiente en el que estamos inmersas. Es fundamental también hablar del tiempo y los espacios necesarios para el autocuidado de cada individuo ya que es imposible tener una vida saludable y poder cuidar a otras personas si no podemos cuidarnos a nosotras mismas. Por último, recalcar que tiene que existir una responsabilidad social de los cuidados y que esto pasa por que los cuidados tengan un lugar central en nuestra sociedad y no un lugar marginal como hasta ahora. Como señalan Carrasco, Borderias y Torns (2011) la organización social de los trabajos de cuidados y el lugar que ocupan en la sociedad actual son producto de un largo proceso histórico que comenzó a gestarse durante la transición al capitalismo liberal. Es fundamental revertir la posición que tienen socialmente y para ello es imprescindible reconfigurar los espacios y los tiempos de la ciudad, pensándolos para poder desarrollar todas las actividades de cuidados.

La dependencia es un concepto construido socialmente, que identifica como tales a grupos de población por razones de edad o de salud; sin embargo, es algo inherente a la condición humana. Todas las personas son social y humanamente interdependientes y requieren distintos cuidados a lo largo del ciclo vital, y el tipo de las dependencias también van variando y pueden ser biológicas, económicas o emocionales. Al ser la dependencia algo universal, los cuidados son inevitables, por lo que su responsabilidad no debería ser individual y privada, sino social y política. (Carrasco, Borderías y Torns, 2011). Cada sociedad debería organizar los cuidados para dar respuesta a las dependencias y necesidades humanas, y a la vez, mantener el respeto por las personas que lo necesitan y no explotar a las que están actuando de cuidadoras (Nussbaum, 2006).

La perspectiva feminista pone en valor las tareas reproductivas y de cuidados, reconoce su impacto cuantitativo y cualitativo en la sociedad 
y visibiliza que actualmente siguen siendo las mujeres quienes desempeñan mayoritariamente las tareas asociadas con lo reproductivo. Sin embargo, cuando se reivindica incorporar las necesidades derivadas de la esfera reproductiva a la planificación urbana no se trata en ningún caso de hacer una "naturalización" de la división sexual del trabajo. Atender a las necesidades relacionadas con lo reproductivo no se traduce en una sectorización sexuada de los espacios sino en preparar el espacio para una sociedad más justa y equitativa en donde las obligaciones que hoy siguen asumiendo mayoritariamente las mujeres sean compartidas (Campos, 1996).

El planificar la ciudad desde una supuesta perspectiva neutra en realidad se basa en las necesidades masculinas priorizando las actividades vinculadas con lo productivo y lo monetario, frente a una invisibilización de las actividades vinculadas con la reproducción y los cuidados. Esto se materializa en las ciudades en aspectos como la localización de los espacios, su diseño, la planificación de la movilidad, la gestión y el mantenimiento de los espacios o cómo se estructuran los horarios.

El objetivo del urbanismo feminista es conseguir cambiar los parámetros sociales que actualmente valoran más las actividades productivas que las reproductivas, para que cada persona pueda elegir qué actividades desarrollar sin que éstas sean definidas por su género, y que la planificación urbana responda a las necesidades derivadas de la esfera reproductiva y los cuidados a través de la configuración urbana.

Abordar la gestión de la vida cotidiana y de los cuidados desde el urbanismo permite obtener una perspectiva más integral de los procesos y la dinámica de la ciudad, considerando las necesidades de la vida diaria de la mayoría de mujeres en relación con la planificación urbana en sus diferentes escalas: desde las grandes intervenciones a nivel central hasta los barrios o conjuntos de viviendas situados en la periferia (Segovia y Rico, 2017).

\section{Un nuevo paradigma urbano: la ciudad cuidadora}

Los entornos urbanos son el escenario en el que desarrollamos nuestras vidas cotidianas, en una estructura urbana que está definida sobre la base de los valores de una sociedad capitalista y patriarcal y en la que ambos sistemas se retroalimentan. Como consecuencia de la dominación patriarcal y de su influencia en la producción del espacio, determinadas actividades son consideradas socialmente más importantes. Esto se materializa en una configuración urbana que jerarquiza actividades y usos, haciendo prevalecer unos frente a otros dedicándoles más espacio, mejores localizaciones y conectividad. El espacio urbano no se ha concebido como espacio donde se desarrollan los cuidados y esto ha llevado a que no se piense la ciudad como soporte físico que facilite la realización de las tareas de cuidados.

Como señala Campos (1996), dar respuesta a las necesidades relacionadas con lo reproductivo no se traduce en una sectorización sexuada de los espacios sino en preparar el espacio para una sociedad más justa y equitativa donde las obligaciones, que hoy siguen asumiendo mayoritariamente las mujeres, sean compartidas.

En esta ciudad social y económicamente injusta las características sociales como el género, la clase social, el ser una persona racializada, la identidad sexual, la diversidad funcional o la edad, entre otros aspec- 
tos, determinan los privilegios y las opresiones que experimentamos en nuestro día a día en el espacio urbano.

La ciudad que tenemos es la materialización territorial de un modelo social y económicamente injusto, por lo que para acabar con las desigualdades sociales y económicas es imprescindible un cambio estructural de paradigma.

Olga Segovia y María Nieves Rico (2017) señalan que aproximarse a un nuevo paradigma urbano implica reconocer la diversidad y dar cuenta de la multiplicidad de caras y habitantes que tiene la ciudad, incorporando a la producción social del hábitat todas las realidades urbanas, incluyendo en particular los derechos de las mujeres en relación con la ciudad y desde una mirada interseccional.

El urbanismo feminista reivindica la importancia social de los cuidados sin que esto signifique encasillar a las mujeres en el rol de cuidadoras, sino asumiendo que todas las personas somos dependientes unas de otras y del entorno y que, por lo tanto, los cuidados deben ser una responsabilidad colectiva. Repensar la ciudad desde una perspectiva feminista es dejar de generar espacios desde una lógica productivista, social y políticamente restrictiva, y empezar a pensar en entornos que prioricen a las personas que los van a utilizar. Para ello se propone un cambio radical de prioridades a la hora de concebir los espacios y los tiempos en la ciudad y construir un nuevo paradigma urbano.

Este nuevo modelo urbano sitúa a las personas en el centro de las decisiones, teniendo en cuenta la diversidad de experiencias y rompiendo con la estandarización de sujetos, cuerpos, vivencias y deseos. Los espacios deben ser flexibles y adaptarse a las diferentes necesidades de las personas y no que las personas se adapten a las condiciones del espacio. Este nuevo paradigma urbano se concreta en el modelo de la ciudad cuidadora, pensando ciudades que nos cuiden, que cuiden nuestro entorno, nos dejen cuidarnos y nos permitan cuidar a otras personas.

En una ciudad que cuida los espacios públicos, las personas perciben seguridad de los espacios, porque están bien señalizados e iluminados; hay gente alrededor que pueda ayudarte; son visibles sin elementos que obstruyan el paso o la visión de las personas; vitales, porque permiten el uso y desarrollo de diferentes actividades y promueven el apoyo mutuo. Cada espacio está pensado desde la vivencia de las personas que lo van a utilizar y cuidando las condiciones físicas y el mantenimiento y gestión del espacio para que cualquier persona puede caminar tranquila a cualquier hora del día sin temor a que la acosen o la agredan.

En este modelo urbano no existe un dominio de los vehículos motorizados que hacen un uso abusivo de los espacios públicos y que producen altos índices de contaminación, accidentes e inseguridad vial, especialmente para las personas mayores y los niños y niñas. Además, Carme Valls-Llobet (2018) nos indica que los efectos de la contaminación ambiental son más negativos en el cuerpo de las mujeres porque muchos contaminantes ambientales actúan como disruptores endocrinos en su organismo. La ciudad que cuida prioriza y fomenta una red de transporte público accesible, física y económicamente, tanto en las estaciones de transporte como en los vehículos y está conectada con una amplia red peatonal y con diferentes espacios (productivos, reproductivos, espacios de ocio, deporte...). Debería haber una variedad de franjas horarias para facilitar los distintos desplazamientos en la vida cotidiana de las personas y sin obligar a invertir una parte considerable de la jornada en desplazamientos. 
Una ciudad que cuida no expulsa a las vecinas de sus barrios por contratos de alquiler abusivos, por la especulación y por regulaciones que solo velan por la propiedad, sino que permite acceder a una vivienda digna en condiciones económicas justas y promueve diferentes modelos de habitar más allá de la convivencia de la familia nuclear heteropatriarcal.

Un paradigma urbano que tiene en cuenta la diversidad y los cuidados asume que las personas somos funcionalmente diversas, que a veces estamos enfermas, tenemos dolores crónicos y que pasamos por diferentes etapas en el ciclo vital que hacen que no encajemos con unos ritmos y niveles de productividad impuestos y que generan frustraciones, miedos y merman nuestra autonomía a la hora de disfrutar de la ciudad.

La ciudad que cuida nuestro entorno no consume recursos territoriales, energéticos y ambientales sin límite. Intenta minimizar los residuos que produce y promueve acciones para limpiar el aire que nos contamina y el agua. Impulsa estrategias para el aprovechamiento de los recursos existentes, por ejemplo, utilizando equipamientos y espacios infrautilizados y priorizando la rehabilitación de edificios y espacios frente a la práctica de la tabula rasa, tan frecuente en urbanismo. Fomenta la distribución equitativa de servicios, equipamientos y comercios de proximidad en los diferentes barrios, lo que da lugar a recorridos funcionales y minimiza el uso del vehículo privado. La ciudad que se preocupa por el entorno construye corredores verdes y desarrolla estrategias para recuperar la flora y la fauna autóctonas.

Una ciudad que permite a las personas cuidarse proporciona espacios equipados para el ocio y la diversidad de prácticas deportivas, y favorece las relaciones interpersonales en espacios públicos exteriores o a salvo de las inclemencias meteorológicas, donde estar, sentarse, charlar y relacionarse, todo ello sin necesidad de mediación de ninguna actividad comercial. Esta ciudad también ofrece espacios para la participación política, libres de la instrumentalización de los entes políticos.

Una ciudad cuidadora también te permite cuidar porque te proporciona el soporte físico necesario para el desarrollo de las tareas correspondientes, como hacer la compra, llevar a niños y niñas al colegio, acompañar a personas enfermas al centro de salud... Este soporte físico se concreta en espacios públicos con juegos infantiles para diferentes edades, con fuentes, baños públicos, vegetación, sombra, bancos y mesas y otros elementos, así como con equipamientos y servicios próximos que facilitan las actividades. La ciudad cuidadora favorece la autonomía de las personas dependientes y, además, permite conciliar las diferentes esferas de la vida cotidiana.

\section{Conclusiones}

Tenemos una sociedad socialmente heterogénea y cada vez más crítica con los modelos de vida a los que nos aboca la ciudad. Sin tiempo para el ocio y para disfrutar de las cosas que nos gustan; envueltas en elementos contaminantes; con espacios que dificultan la conciliación de tareas y donde los cuidados están invisibilizados en el espacio urbano; donde las personas que no se adaptan a los ritmos productivos del capitalismo salvaje son excluidas sistemáticamente y se les niega el derecho a la ciudad; donde las mujeres somos acosadas y agredidas en los espacios urbanos como algo cotidiano; con modelos de movilidad que 
siguen priorizando el uso del vehículo privado y con redes viarias que priorizan el desarrollo de las actividades productivas...

Ante esta realidad es urgente un cambio de paradigma urbano para que todas las personas podamos satisfacer nuestras necesidades en la ciudad y donde los cuidados y la sostenibilidad de la vida estén en el centro de las decisiones urbanas. 


\section{Referencias}

Agenjo, A. (2013). Sostener la vida: respuestas feministas en torno a la organización social de los cuidados. En Vicent, L., Castro, C., Agenjo, A. et al., El desigual impacto de la crisis sobre las mujeres. Madrid: Dossier FUHEM ecosocial, 22-28. Recuperado el 13 de agosto de 2018, de: http:/ / www.fuhem.es/ media/cdv/file/biblioteca/Dossier/dossier_El-desigual-impacto-de-la-crisis-sobre-las-mujeres.pdf.

Beall, J. (1996). Participation in the city: where do women fit in? Gender and Development, 4(1), 9-16. DOI: https://doi.org/10.1080/741921946.

Bondi, L. y Rose, D. (2003). Constructing gender, constructing the urban: A review of Anglo-American feminist urban geography. Gender, Place $\mathcal{E}^{2} \mathrm{Cul}$ ture: A Journal of Feminist Geography, 10(3), 229-245. DOI: https://doi.org/10.1080/0966369032000114 000.

Bosch, A., Carrasco, C. y Grau, E. (2005). Verde que te quiero violeta. Encuentros y desencuentros entre feminismo y ecologismo. En Tello, E. (ed.), La historia cuenta. Del crecimiento económico al desarrollo humano sostenible. Barcelona: Fundació Nous Horizons | El Viejo Topo, 321-346.

Bourdieu, P. (2000). La dominación masculina. Barcelona: Anagrama, 2000.

Brullet, C. (2010). Temps, cura i ciutadania. Corresponsabilitats privades i publiques. Barcelona: Programa Nous Usos Socials del Temps-Ajuntament de Barcelona.

Campos, P. (1996). Influencia de las ciudades en la vida de las mujeres. En Rubio Alférez, C. y Ardid Gumiel, M. (eds.), Mujer y Urbanismo: Una recreación del espacio. Claves para pensar en la ciudad y el urbanismo desde una perspectiva de género. Madrid: Federación Española de Municipios y Provincias (FEMP) | Instituto de la Mujer Ministerio de Asuntos Sociales, 23-30.

Carrasco Bengoa, C. (2007). Estadístiques sota sospita: proposta de nous indicadors des de l'experiència femenina. Barcelona: Generalitat de Catalunya - Institut Català de les Dones. Recuperado el 18 de agosto de 2018, de: http://dones.gencat.cat/web/.content/03_ambits/docs/publicacions_eines07.pdf.

Carrasco, C., Borderías, C. y Torns, T. (2011). Introducción. El trabajo de cuidados: antecedentes históricos y debates actuales. En Carrasco, C., Borderías, C. y Torns, T. (eds.), El trabajo de cuidados: historia, teoría y políticas. Madrid: Catarata. Recuperado el 13 de agosto de 2018, de: https://www.fuhem.es/media/cdv/file/biblioteca/Economia_critica/El-trabajo-de-cuidados_introduccion.pdf.

Darke, J. (1998a). La ciudad, espacio de propiedad patriarcal. En Booth, C., Darke, J. y Yeandle, S. (eds.),
La vida de las mujeres en las ciudades: la ciudad, un espacio para el cambio. Madrid: Narcea Ediciones, 117121.

Darke, J. (1998b). La ciudad zonificada: estereotipos de género. En Booth, C., Darke, J. y Yeandle, S. (eds.), La vida de las mujeres en las ciudades: la ciudad, un espacio para el cambio. Madrid: Narcea Ediciones, 122-126.

Del Valle, T. (1996). Incidencia de las nuevas socializaciones en la elaboración de la memoria. En González Echevarría, A., Epistemología y Método. VII Congreso de Antropología, Zaragoza, España.

Durán, M.A. (1998). La ciudad compartida. Conocimiento, afecto y uso. Madrid: Consejo Superior de los colegios de arquitectos de España.

Durán, M. A. (2000). Si Aristóteles levantara la cabeza. Madrid: Ediciones Cátedra.

England, K. (1991). Gender relations and the spatial structure of the city. Geoforum, 22(2), 135-147. DOI: https://doi.org/10.1016/0016-7185(91)90003-9.

Fernández Moreno, N. (1995). Una aproximación antropológica al origen de los espacios segregados. En Bisquert Santiago, A. (ed.), Actas de curso: Urbanismo y mujer. Nuevas visiones del espacio público y privado, Málaga 1993-Toledo 1994. Madrid: Seminario permanente Ciudad y Mujer, 99-106.

Friedan, B. (1963). The Feminine Mystique. New York: W.W. Norton.

García Ramón, M. D. (1989). Para no excluir del estudio a la mitad del género humano. Un desafío pendiente en geografía humana. Boletín de la Asociación de Geógrafos españoles, 9, 27-48.

Greed, C. (1997). Género y planificación del territorio ¿Un mismo tema? En Forúm Internacional de planificación del territorio desde una perspectiva de género, Barcelona, Fundació Maria Aurèlia Capmany.

Grupo de Ecofeminismo-Ecologistas en Accion (2011). Menos para vivir mejor. Ecofeminismos, anticapitalismo y mundo urbano [archivo PDF]. Recuperado el 13 de agosto de 2018, de: http:/ / www.mundubat.org/wp-content/uploads/archivos/201303/ menos-vivir-mejor-final_grupo-ecofeminismo.pdf.

Hayden, D. (1982). The grand domestic revolution: A history of feminist designs for American homes, neighborhoods, and cities. Cambridge: the MIT Press.

Herrero, Y. (2017). Economía ecológica y economía feminista: Un diálogo necesario. En Carrasco Bengoa, C. y Díaz Corral, C. (eds.), Economía feminista: desafios, propuestas y alianzas. n.a.: Entrepueblos/Entrepobles/Entrepobos/Herriarte, 121-142.

Jaeckel, M. y Van Geldermalsen, M. (2006). Planificació urbanística amb enfocament de gènere. En Diputació de Barcelona, Urbanisme i gènere. Una visió 
necessària per a tothom. Barcelona: Diputació de Barcelona -Xarxa de municipis, pp. 99-108. Recuperado el 13 de agosto de 2018, de: https://www1.diba. cat/uliep/pdf/36241.pdf.

Larsson, A. (2006). From equal opportunities to gender awareness in strategic spatial planning: Reflections based on Swedish experiences. Town Planning Review, 77(5), 507-530.

Levy, C. (1996). The process of Institutionalising Gender in Policy and Planning: the web of institutionalization. DPU Working Paper n. ${ }^{\circ}$ 74, London: DPUUniversity College London. Recuperado el 18 de agosto de 2018, de: https://www.ucl.ac.uk/bartlett/development/sites/bartlett/files/migrated-files/wp74_0.pdf.

Levy, C. (2003). Ciudad y género. Una ciudad más justa: el género y la planificación. En Balbo, M, Jordán, R. y Simioni, D. (eds.), La Ciudad Inclusiva. Santiago de Chile: Naciones Unidas | CEPAL (Cuadernos de la CEPAL n. ${ }^{\circ} 88$ ), 237-258. Recuperado el 18 de agosto de 2018 , de: https://repositorio.cepal.org/bitstream/handle/11362/27814/ S2003002_es.pdf;jsessionid=8341F69732A246CA61 1D816D59208F4A?sequence=1.

Llop, M. (1996). La ciudad para su uso. En Rubio Alférez, C. y Ardid Gumiel, M. (eds.), Mujer y Urbanismo: Una recreación del espacio. Claves para pensar en la ciudad y el urbanismo desde una perspectiva de género. Madrid: Federación Española de Municipios y Provincias (FEMP) | Instituto de la Mujer Ministerio de Asuntos Sociales.

Massolo, A. (2005, junio). Género y Seguridad Ciudadana: el papel y reto de los gobiernos locales. En PNUD El Salvador (Programa de las Naciones Unidas para el Desarrollo), Programa 'Hacia la Construcción de una sociedad sin Violencia'. Ponencia en el Seminario Permanente sobre Violencia, El Salvador. Recuperado el 18 de agosto de 2018, de: http:/ / docs.wixstatic.com/ugd/ef40bd_101e48ef3e0e4b8 da7fd2d2a70356969.pdf.

McDowell, L. (1999). Género, identidad y lugar. Un estudio de las geografías feministas. Madrid: Ediciones Cátedra.

Miralles-Guasch, C. (2010). Dones, mobilitat, temps i ciutats. Barcelona: Generalitat de Catalunya - Institut Català de les Dones (Colección Quaderns de l'Institut 14, "Perspectives des del feminisme"). Recuperado el 08 de agosto de 2018, de: http:/ / dones.gencat.cat/web/.content/03_ambits/docs/publicacions_quaderns14.pdf.

Moser, C. y Levy, C. (1986). A theory and methodology of gender planning: meeting women's practical and strategic needs. London: Development Planning Unit (DPU)DPU, University College London.

Moser, C. (1989). Gender planning in the Third World: Meeting practical and strategic gender needs. World Development, 17(11), 1799-1825. DOI: https://doi.org/10.1016/0305-750X(89)90201-5.
Murillo, S. (1996). El mito de la vida privada. Madrid: Siglo XXI.

Muxí Martínez, Z. (2009). Recomanacions per a un habitatge no jeràrquic ni androcèntric. Barcelona: Institut Català de les Dones / Generalitat de Catalunya-Departament de Medi Ambient i Habitatge. Recuperado el 13 de agosto de 2018, de: https://punt6.files. wordpress.com/2011/03/recomanacionsperaunhabitatgenojerc3a0rquicniandrocentric2.pdf.

Muxí Martínez, Z. (2013). Postsuburbia: después del espejismo. En: Muxí, Z (ed.) Postsuburbia: rehabilitación de urbanizaciones residenciales monofuncionales de baja densidad. Barcelona: Comanegra.

Nussbaum, M. (2006). Poverty and Human Functioning: Capabilities as fundamental entitlements. En Grusky, D.B. y Kanbur, R. (eds.), Poverty and inequality. Stanford: Stanford University Press, 47-75.

Pérez Fernández, I. (2009). Espacio, identidad y género. Sevilla: Arcibel Editores.

Pérez Orozco, A. (2014). Subversión feminista de la economí: aportes para un debate sobre el conflicto capitalvida. Madrid: Traficantes de sueños. Recuperado el 18 de agosto de 2018, de: https://www.traficantes. net/sites/default/files/pdfs/map40_subversion_feminista.pdf.

Picchio, A. (2009). Condiciones de vida: Perspectivas, análisis económico y políticas públicas. Revista de Economía Crítica, 7, 27-54. Recuperado el 18 de agosto de 2018, de: https://ideas.repec.org/a/ret/ ecocri/rec07_02.html.

Rainero, L. y Rodigou, M. (2001). Indicadores urbanos de género. Instrumentos para la gobernabilidad urbana. Córdoba, Argentina: CICSA.

Ryan, M. (1990). Women in public: Between banners and ballots, 1825-1880. Baltimore: Johns Hopkins University Press.

Sabaté, A., Rodríguez J. y Díaz, M.A. (1995). Mujeres, Espacio y Sociedad. Hacia una Geografía del género. Madrid: Síntesis.

Sáinz Guerra, J. L. (2004). Los cambios en la vivienda de la ex-República Democrática Alemana a partir de la reunificación. Ciudades, 8, 63-85. Recuperado el 18 de agosto de 2018, de: http://www3.uva. es/iuu/REVISTA/Ciudades \% 2008/Ciudades \% 20 08\% 20063-085\% 20SAINZ\% 20GUERRA.pdf.

Sánchez de Madariaga, I. (2004). Infraestructuras para la vida cotidiana y calidad de vida. Ciudades, 8 , 101-133. Recuperado el 13 de agosto de 2018, de: http://www3.uva.es/iuu/REVISTA/Ciudades $\% 20$ 08/Ciudades $\%$ 2008\% 20101-133\% 20SANCHEZ\% 20 MADARIAGA.pdf.

Sandercock, L. y Forsyth, A. (1992). A gender agenda: new directions for planning theory. Journal of the American Planning Association, 58(1), 49-59. DOI: https://doi.org/10.1080/01944369208975534.

Segovia, O. y Rico, M. N. (2017). ¿Cómo vivimos la ciudad? Hacia un nuevo paradigma urbano para la igualdad de género. En Rico, M. N. y Segovia, O. 
(eds.) ¿Quién cuida en la ciudad? Aportes para políticas urbanas de igualdad. Santiago de Chile: Naciones Unidas - Comisión Económica para América Latina y el Caribe (CEPAL), 41-69.

Segura, I. (2006). La memoria, una forma de arquitectura. En Diputació de Barcelona, Urbanisme i gènere. Una visió necessària per a tothom. Barcelona: Diputació de Barcelona -Xarxa de municipis, 29-32. Recuperado el 13 de agosto de 2018, de: https://www1. diba.cat/uliep/pdf/36241.pdf.

Soto Villagrán, P. (2007). Ciudad, ciudadanía y género. Problemas y paradojas. Territorios, 16-17, 29-45. Recuperado el 18 de agosto de 2018, de: https:/ / revistas.urosario.edu.co/index.php/territorios/article/view/839.

Spain, D. (2006). La importancia de los espacios de género urbanos en el ámbito público. En Diputació de Barcelona, Urbanisme i gènere. Una visió necessària per a tothom. Barcelona: Diputació de Barcelona -Xarxa de municipis, 33-42. Recuperado el 13 de agosto de 2018, de: https://www1.diba.cat/uliep/ pdf/36241.pdf.

Torns, T., Borras, V., Moreno, S., et al. (2006). Les politiques de temps: un debat obert. Barcelona: Ajuntament de Barcelona-Regidoria de Nous Usos del Temps. Recuperado el 18 de agosto de 2018, de: http:/ / ajuntament.barcelona.cat/tempsicures/sites/default/files $/ 1 \% 20$ Les $\% 20$ politiques $\% 20 \mathrm{del} \% 20$ temps \%20un\%20debat\%20obert.pdf.
Tronto, J. (2005). Cuando la ciudadanía se cuida: una paradoja neoliberal del bienestar y la desigualdad. En Rincón, A., Congreso Internacional Sare 2004: “¿Hacia qué modelo de ciudadanía?”, Bilbao: EMAKUNDE-Instituto Vasco de la Mujer, 231-253. Recuperado el 18 de agosto de 2018, de: http:/ / www.emakunde.euskadi.eus/contenidos/informacion/pub_jornadas/es_emakunde/adjuntos / sare2004_es.pdf.

Valls-Llobet, C. (2018). Medio ambiente y salud. Mujeres y hombres en un mundo de nuevos riesgos. Madrid: Ediciones Cátedra.

Velázquez, I. (2006). Una mirada atrás: Mujeres en el urbanismo y en la construcción de la ciudad. En Diputació de Barcelona, Urbanisme i gènere. Una visió necessària per a tothom. Barcelona: Diputació de Barcelona -Xarxa de municipis, 17-28. Recuperado el 13 de agosto de 2018, de: https://www1.diba.cat/ uliep/pdf/36241.pdf.

Walker, J., Frediani, A. A. y Trani, J. F. (2013). Gender, difference and urban change: implications for the promotion of well-being. Environment and Urbanization, 25(1), 111-124. DOI: https://doi. org/10.1177/0956247812468996.

Wekerle, G. R. (1980). Women in the urban environment. Signs: Journal of Women in Culture and Society, 5(S3), S188-S214.

Valdivia, B. (2018). Del urbanismo androcéntrico a la ciudad cuidadora. Hábitat y Sociedad, 11, 65-84.

<http://dx.doi.org/10.12795/HabitatySociedad.2018.i11.05> 